\title{
Analysis of Frequency Selective with Nonlinear Antenna under Radiations of Bi-Frequency Waves Based on Genetic Algorithm
}

\author{
Mona Teimoory ${ }^{1}$, Saeed Reza Ostadzadeh ${ }^{2}$, Behrooz Abdoli ${ }^{3}$ \\ ${ }^{1}$ Shahab Danesh University, Qom, Iran \\ daneshjou116@gmail.com \\ ${ }^{2}$ Arak University, Arak, Iran \\ ${ }^{3}$ Shahab Danesh University, Qom, Iran
}

\begin{abstract}
The more frequency radiation increases, the shorter becomes its wave length. The short wavelength, while hitting to some material, cause vibration and make change the positive and negative poles. As the most common way of genetic algorithm codification is using zero and one alphabet, genetic algorithm field is series of potential responses which are codified by some ways. Generally, the primitive population greatness has 30 or 100 members. In this study, genetic algorithm is applied for analyzing frequency selective surfaces with nonlinear antenna, in order to obtain two poles of nonlinear loads. It is worth to mention that two waves, with two different frequencies between 100 and $120 \mathrm{MHz}$, are radiated to antenna.

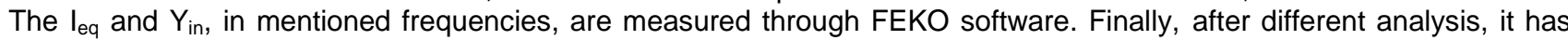
been determined that in order to analyze selective surfaces, like harmonic balance, which are only capable of single frequency radiation, more global genetic algorithm analysis would be introduced for frequency selective surfaces.
\end{abstract}

Key words: genetic algorithm, frequency selector, nonlinear antenna and wave radiation

\section{INTRODUCTION}

Electromagnetic waves with wavelength shorter than radio waves and longer than infrared waves are called microwaves. It seems that microwave wavelengths, like its name, are waves with less than $1 \mathrm{~mm}$ of wavelength, and wavelength of microwaves are placed between $1 \mathrm{~mm}$ (equivalent to $300 \mathrm{GHz}$ frequency) to $30 \mathrm{~cm}$ (equivalent to $1 \mathrm{GHz}$ of frequency). However, there is not a common idea about the limitation of such definition, and sometimes, it is regarded starting from $0.3 \mathrm{~mm}$.

Microwaves or microwaves, are made from combination of micro, meaning small and wave, and they are waves with short wavelength and much vibrations (frequencies). Microwave is a kind of electromagnetic waves, and in fact, they are radio waves with very high frequencies. The more the radiation is high, the shorter would be its wavelength. The frequencies of such waves could be between $300 \mathrm{MHz}$ to several $\mathrm{GHz}$ per second. Coverage of such waves is short, and it is almost several meters, but their infiltration rate is rather high. The more the frequency is, the more is the infiltration rate, but wave range would be shorter (Lee, 2000).

Such wave, while encountering a material, may reflect, radiate, or absorbed. Metal materials reflect fully such waves. Most of nonmetal materials like glass and plastic, conduct such waves through themselves, and materials containing water as meals and even men, absorb such waves. If the energy absorption rate of a material is higher than its loss of energy, temperature of that material will elevate. Waves with low wavelength, while hitting a material, cause such a vibration and change of positive and negative poles, that such high vibration of molecules makes a big disorder among them and create fraction among molecules and finally make that material to get warm (Fang et al., 2014).

Generally, in last half century, microwave and antenna engineering has experienced a significant development, due to development of communication technology, and increasing need to high transfer rate and bandwidth, and the variety of applications, has caused the increasing development of this branch of engineering science. Radio and microwave system world, covers a frequency field from several hundred kilohertz to several hundred gigahertz. With an overview of radio system evolution history, we can predict that high frequency communication technology in 21 century will continue with a high rate of development to its growth, and effecting on man's life. Accordingly, being familiar with microwave and antenna principals seems vital for engineers and all those who are involved in radio systems.

\section{HARMONIC BALANCE PRINCIPALS}

Figure 1 shows a general circuit which covers a wide range of nonlinear circuit with high frequency. A circuit is a nonlinear semiconductor piece which is connected to load and big signal source input. At first, we assume that stimulation of input includes a basic frequency (also, every other intermittent entrance) and the fact that DC bias can be integrated in input or output. Adaptive networks at input and output of are applied in entrance and output channel for performance optimization, connection of bias voltage to circuit and also for filter and restrict various harmonics properly. Semiconductor device is often a diode or a transistor, and we have to assume that we can describe a equivalent circuit with two part: one linear, the other nonlinear. The circuits are linear adaptive, and carry out most of the time filtering and transfer of impedance (Razaq, 2015). 


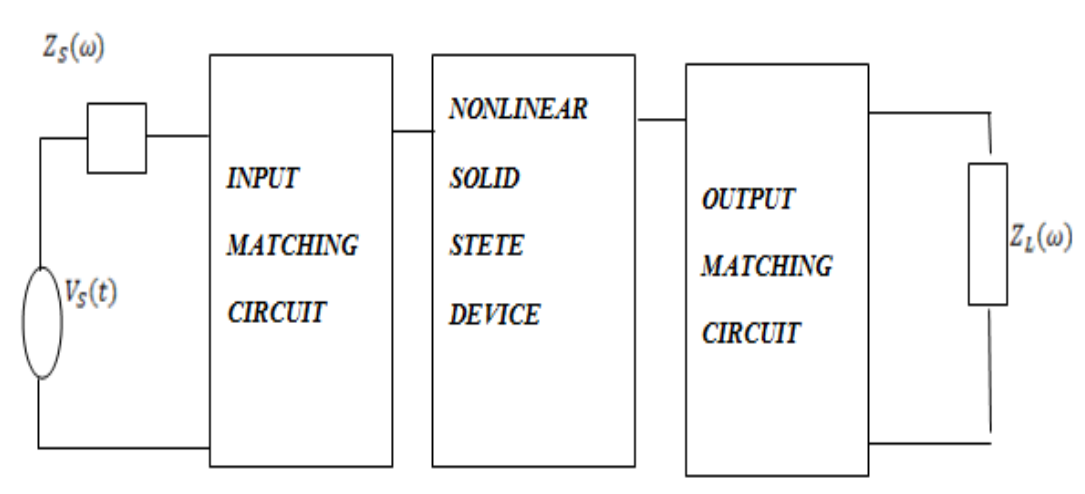

Figure (1) general equivalent circuit in nonlinear two-entrance span

Therefore, the analysis of such circuits doesn't seem so hard. A solution to write a set of differential equations is time field, which describes nonlinear part and adaptive circuits, simultaneously. By solving such equations, we can obtain the stable state voltage, and with Fourier transform, we can determine them, that means, we will have the desired outlet harmonic. Differential equations would be nonlinear, and must be solved numerically. In fact, most of nonlinear circuits are analyzed successfully, using this method.

The elements of Figure (1) can be classified as Figure (2). So, it is transformed to two sub circuits: linear and nonlinear. Linear sub circuit is regarded as one or multi span, which can be described with matrix $Y$ and $S$ or other relevant matrix. Nonlinear elements with I/V or Q/V properties, becomes models, and must be defined in time area. So, the circuit will reduce to $\mathrm{N}+2$ network span, in a way that nonlinear elements are connected to $\mathrm{n}$ port, and voltage sources are connected to other ports $(\mathrm{N}+1$ and $\mathrm{N}+2$ ports are shown as input and outputs. a sinusoidal source is connected solely to one of the ports. However, in Figure 2, the sources are placed in each port in order to maintain the integrity of circuit).

$Z_{s}(\omega)$ and $Z_{L}(\omega)$ are respectively source and load impedance, and are place under linear circuit. They are situated in series circuit, in input and output ports, and in some cases, it is necessary to be recovered. Voltage and current of any port can be described in time or frequency domain. Due to existence of nonlinear elements, voltage and currents of ports must include input stimulation harmonics. Although, the number of voltage and current of each port are infinite, theoretically, in all parts of this research, we intend to assume that voltage and currents can be described by DC and K factors of first harmony, accurately. So, we can conclude that higher harmonies are negligible (Hong et al., 1993).

Circuit in Figure (2) can be regarded as analyzed, whenever we know the coordinates of voltage and steady current in each of ports. This way, knowing the frequency factors in all ports is in continuation of solution, because time area waves shape can be transferred to frequency area, using Fourier transform. If the voltage of a port would be determined in frequency area, we can calculate port current for under linear circuit, using matrix $Y$. In addition, in other hand, we can calculate the voltage in time area, with Fourier reverse transform of port voltage, and then, by help of I/V equation in nonlinear element, we can calculate port current. The idea of harmonic balance is to find out the port set voltage (or voltage harmonic), in order to obtain the port current from linear or nonlinear part of circuit. When we reach to such voltage, in fact we have solved the circuit.

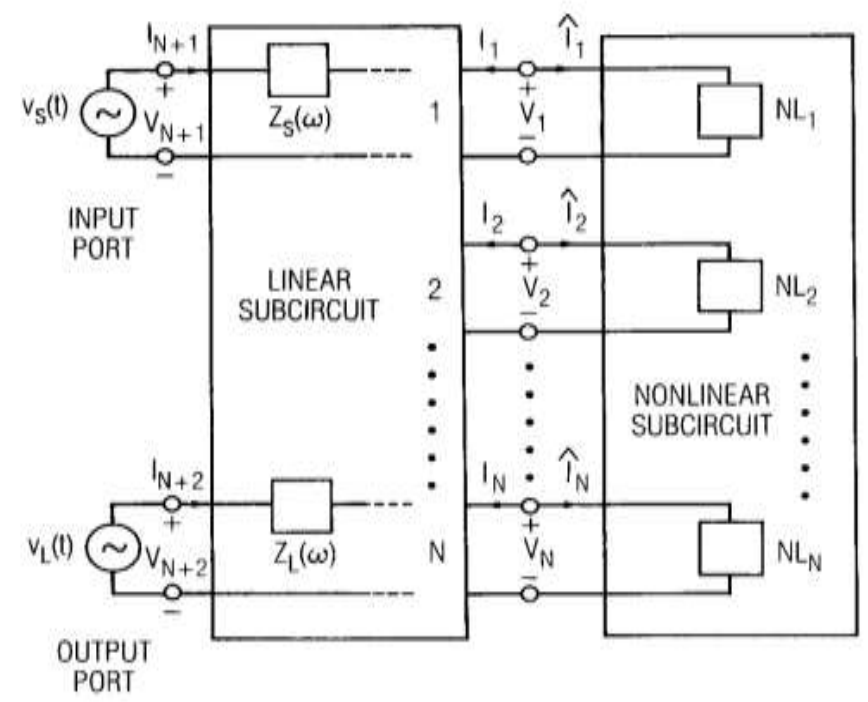

Figure 2: A high frequency nonlinear circuit, divided to linear and nonlinear sub circuit

load resistance and source resistance is integrated in linear part of circuit. 
Newton algorithm is a powerful algorithm to find out the zero of a multi-variant function. As the harmonic balance leads at last hand to finding out the zeros of $\mathrm{F}(\mathrm{V})$ function, therefore, Newton algorithm is an appropriate selection as a solution. Newton method is a repetitive method. This method continues using primary derivative of function and exterpolation on independent variable axis, and the repetition of this process continues, until we find the zero of function. At the same time, it has a desirable accuracy. The strong point of this method is that it uses voltage vector $V$ and all of the derivation of $F(V)$ in relation to $\mathrm{V}$ in all harmonics in each iteration phase, in order to find new guess of $\mathrm{V}$.

According to Newton iteration relation, we have:

$$
\begin{aligned}
& F\left(V^{P}\right)-\frac{\partial F(V)}{\partial V} \mid \Delta V=0 \quad, V=V^{P} \\
& V^{P}-V^{P+1}=\Delta V
\end{aligned}
$$

So, the new value $\mathrm{V}^{\mathrm{P}+1}$ is as follows:

$V^{P+1}=V^{P}-\left(\frac{\partial F(V)}{\partial V}\right)^{-1} F\left(V^{P}\right) \quad, V=V^{P}$

Briefly, we can categorize the harmonic balance relation, using Newton iteration method:

1. We make an initial guess of $v_{1}(t)$.

2. Fourier transform of $v_{1}(t)$ is shown with $V_{1}^{0}$, which is the initial guess in frequency domain. The upper zero is indicator of iteration number.

3. The relations (26-2) and (27-2) is formed in relation to application.

4. Functions $J_{F}$ and $F\left(V^{0}\right)$ are formed.

5. Using relation (27-2), we obtain the value of $V_{1}^{1}$.

6. Using the relation I/V concerning the nonlinear part of circuit, the current I is calculated for $V_{1}^{1}$.

7. We form the $F\left(V^{1}\right)$.

8. If the error is trivial sufficiently, $\mathrm{V}^{1}$ is the answer. Otherwise, we obtain inverse Fourier transform of $\mathrm{V}^{1}$, and repeat the level 3 (Zoo et al., 2010).

The most significant advantage of Newton method is the use of derivative of error function in relation to each variable and each port. Because of this, such method can obtain the convergence with big number of variables, in condition that nonlinear value of circuit would not be very high and the initial guess would be rather good (Mandel and Sarkar,2015).

From the materials presented in this study, we can conclude that harmonic balance method as a frequency domain method has no restriction on load nonlinearity degree, but it has its own disadvantages:

1) Radiation wave must be single frequency.

2) Radiation wave contain low calculation performance.

In order to solve the above issues, recently, the calculations are carried out with genetic algorithm.

\section{GENETIC ALGORITHM}

Genetic algorithm is probability research and optimization methods, which are made out of concepts adopted from natural biologic evolutions like reproduction, coupling and mutation. Generally, genetic algorithms belong to a bigger category of evolutionary algorithm including evolutionary program, evolutionary strategies and genetic programming. Evolutionary algorithm for an issue starts with a population of potential answers, then, this population is placed under survival of the fittest, and by reproduction and mutation operation, every time, more fitting population is created, and gradually we can reach to better approximation of optimized answers. In every stage of iteration, evolutionary algorithm creates new population of approximations, by selection and iteration process. Created populations became more and more compatible with their environment and the considered domain of the issue, and this action is exactly similar to the process which occur in natural compatibility (Gousouami and Mendel, 2013).

Generally, genetic algorithms are constituted from following components:

\section{- Chromosom}

In genetic algorithms, every chromosome is indicator of a point in research space, and a possible solution for relevant issue. Chromosomes (solutions), themselves, are formed from a fixed number of genes (variable). In order to show the chromosomes, usually binary codification (bit series) is used.

\section{- Population}

A set of chromosomes create a population. By the influence of genetic operators on every population, a new population is created with the same number of chromosomes. 


\section{- Fitness Function}

In order to solve a problem, using genetic algorithm, we must invent a fitness function for that problem. For every chromosome, this function, gives us a none-negative value, which is indicator of fitness or individual capability of that chromosome.

\section{- Genetic Actuator}

All communication and electronic device, which operate in special frequency domain use filters inside their circuit structure, for eliminate other available frequencies. Depending on the type of favorable electronic devices, the existing filters are categorized in three types: high-pass filter, low-pass filter, band-pass filter or composite. However, each of desired filters shows the expected performance, in specific frequency area. When the frequency is increased to gigahertz and terahertz level, significantly, the other capacitors and inductors forming these filters loss their inherent property, and so the natural filters can't be used in this frequency range. In order to solve filtering problem in high frequency, frequency selective surfaces (FSS) are addressed since 1960. Research method used in this study is simulation, and using FSS software, we will analyze the issues. At the beginning, single antenna and then antenna array is used in order to achieve the desired goal.

\section{SIMULATION AND RESULTS}

\subsection{Single Antenna Results}

As we have already mentioned, frequency selection surfaces are used in radar systems abundantly. That means if two waves with two frequencies, for example $f_{1}$ and $f_{2}$ radiated to nonlinear antenna, it is ideal that returning wave contains determined harmony. In order to receive special harmonies, we use antenna. In figure below, we can see the image of a dipole antenna, which is under the radiation of exterior wave:

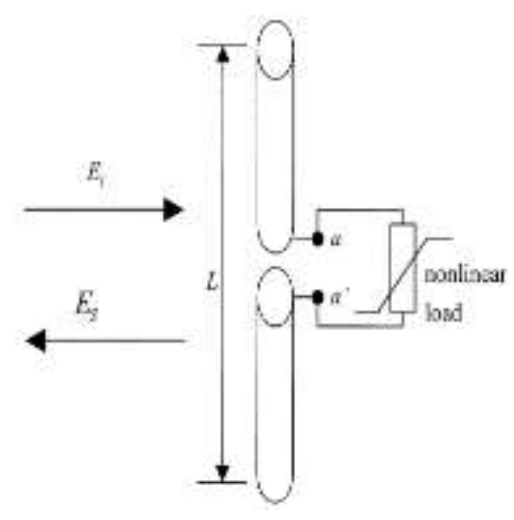

Figure (3): nonlinear dipole antenna

Analysis of such issues is in such a way that Norton equivalent circuit of nonlinear antenna are used. In following figure, electronic equivalent circuit of nonlinear dipole antenna is shown:

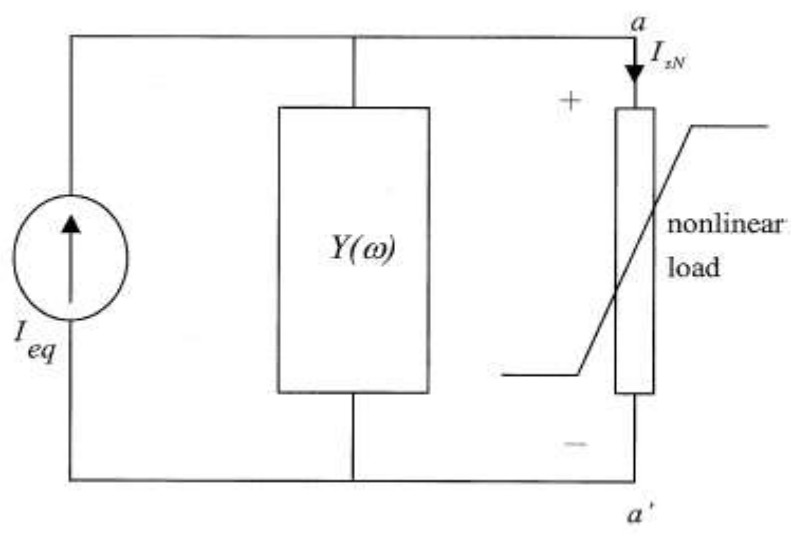

Figure (4): high frequency electronic equivalent circuit, connected to nonlinear load

Due to restrictions of harmonic balance analysis, for example, being single frequency of radiation wave and low calculation performance in recent years, researchers were looking for optimization of this method. One of the conventional algorithms for optimization is genetic algorithm. 
In this research, genetic algorithm is used for analysis of frequency selective surface with nonlinear antenna, in order to obtain two poles of nonlinear load.

In our equivalent circuit (figure 3), leq current, will take a value as follows, for single antenna:

$$
I_{e q}(t) \approx \sum_{i=1}^{M}\left\{I_{i} \sin \left(\omega_{i} t+\phi_{i}\right)\right\}
$$

equation (1)

In this relation, $\mathrm{M}$ is indicator of the number of sinusoidal harmonics for $\mathrm{I}_{\text {eq. }}$.

It is worth to mention, two waves are radiated with 100 and $120 \mathrm{MHz}$ of frequency to antenna. $\mathrm{l}_{\text {eq }}$ and $Y_{\text {in }}$ values mentioned frequencies are calculated through FEKO software. Table below shows such values:

Table 1: $I_{\text {eq }}$ and $Y_{\text {in }}$ values in input frequencies

\begin{tabular}{|c|c|c|}
\hline$f$ & 100 & 120 \\
\hline$Y_{\text {in }}$ & & \\
\hline$I_{\text {eq }}$ & & \\
\hline
\end{tabular}

In this research, we have used following relation for nonlinear load:

$i=\frac{1}{75} v+4 v^{3}$

equation (2)

Now, by using $\mathrm{kcl}$ in node a, we can write:

$$
K C L(a) \Rightarrow I_{e q}-I^{\prime}{ }_{i n}-I_{S N}=0 \quad \text { equation (3) }
$$

The number of created frequency harmonics in nonlinear load consists of linear combination of received frequency, so we have:

$$
\omega_{j}=k_{1} \omega_{1}+k_{2} \omega_{2}+\ldots . . k_{M} \omega_{M}, \quad j=1,2, \ldots . N
$$

equation (4)

Here $\mathrm{k}_{\mathrm{i}}, \mathbf{i}=1,2, . . \mathrm{M}$, and considering the nonlinear load degree of circuit, $\sum_{i=1}^{M}\left|\boldsymbol{k}_{\boldsymbol{i}}\right| \leq 3$. Created frequency harmonic values, are shown in following tables:

\begin{tabular}{|c|c|c|c|c|c|c|c|}
\hline$K_{2} K_{1}$ & -3 & -2 & -1 & 0 & 1 & 2 & 3 \\
\hline-3 & & & & 360 & & & \\
\hline-2 & & & $\times$ & $\times$ & $\times$ & & \\
\hline-1 & & $\times$ & $\times$ & $\times$ & $\times$ & 80 & \\
\hline 0 & $\times$ & $\times$ & $\times$ & 0 & 100 & 200 & 300 \\
\hline 1 & & $\times$ & 20 & 120 & 220 & 320 & \\
\hline 2 & & & 140 & 240 & 340 & & \\
\hline 3 & & & & 360 & & & \\
\hline
\end{tabular}

Table (2): created harmonic frequency

Generally, after applying genetic algorithm for solving problem, the results are obtained as follows. 


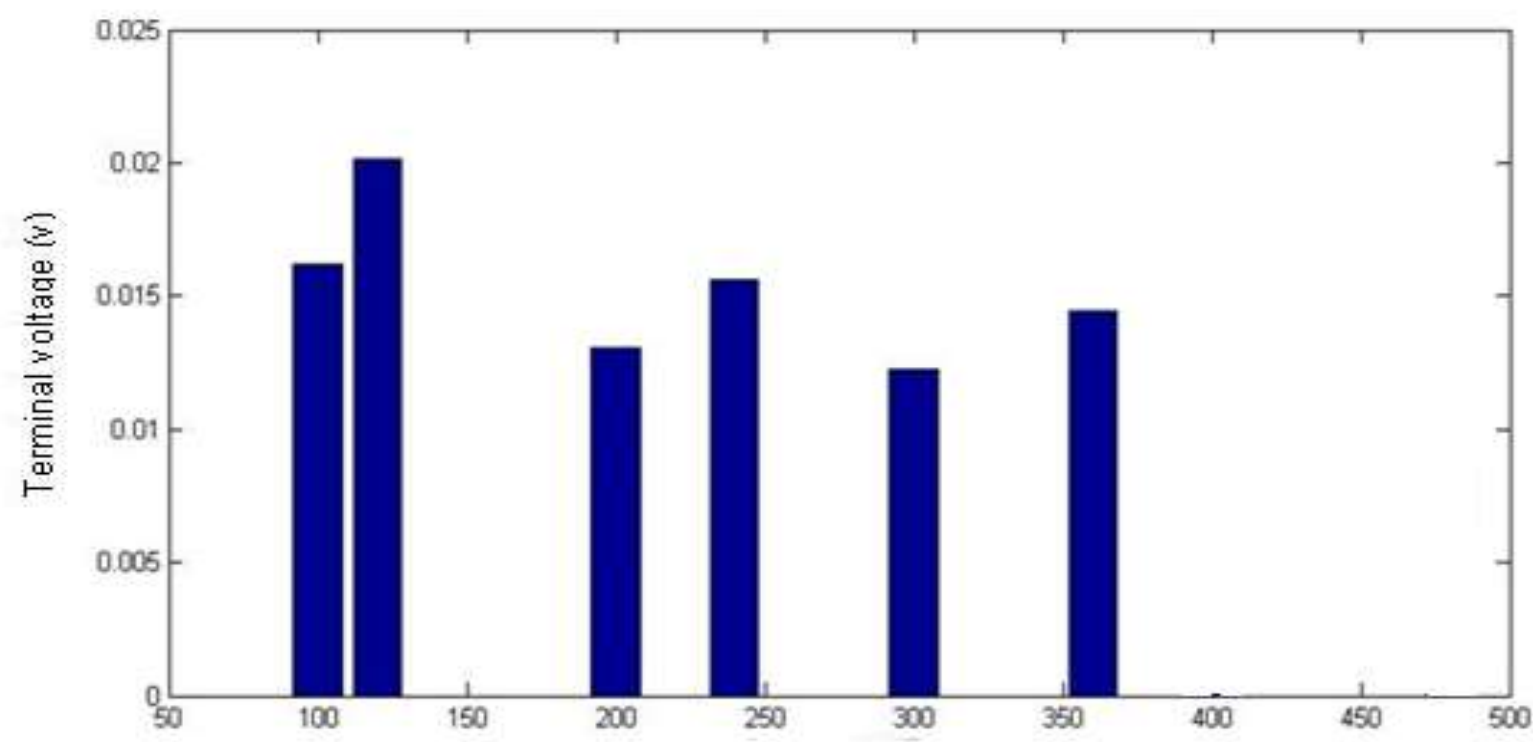

frequency $(\mathrm{MHz})$

Figure 5: voltage values of nonlinear load poles in different frequencies

Voltage diagram is according to frequency, for two input frequency 100 and 120. Comparing figure 5 and table 2 we can understand that voltage domain in some created frequencies is calculated zero, after applying genetic algorithm. In continuance, we will address analysis of antenna array, in order to eliminate or attenuation of frequency harmonics.

It is worth mentioning that convergence factor for genetic algorithm 0.0001 and the number of algorithm iteration is considered 1000.

\subsection{Output for Antenna Array:}

After designing an output for single antenna, the same action is carried out for antenna array. The process of voltage calculation of two poles in antenna array is similar to single antenna, with the only difference that in antenna array, instead of previous admittance matrix, we will use new admittance matrix. In other words, in antenna array, also, calculations are like single antennas, and the only difference is in admittance matrix, which is mentioned in as follows:

In following figure, an antenna array is shown:

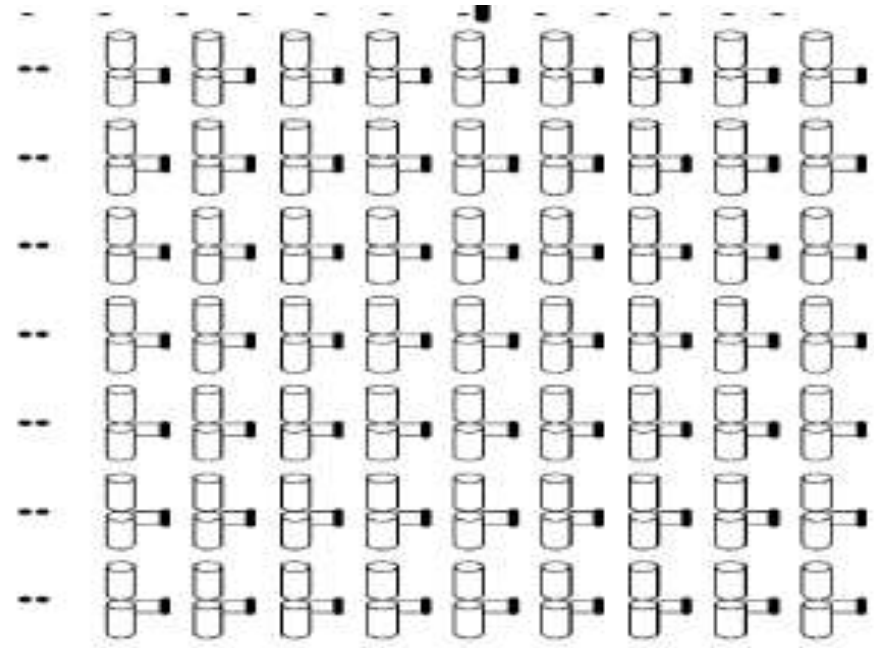

Figure 6: nonlinear dipole antenna array 
As in single antenna analysis, we have used equivalent circuit, in antenna array, we will use equivalent circuit. Following figure shows equivalent circuit of antenna array:

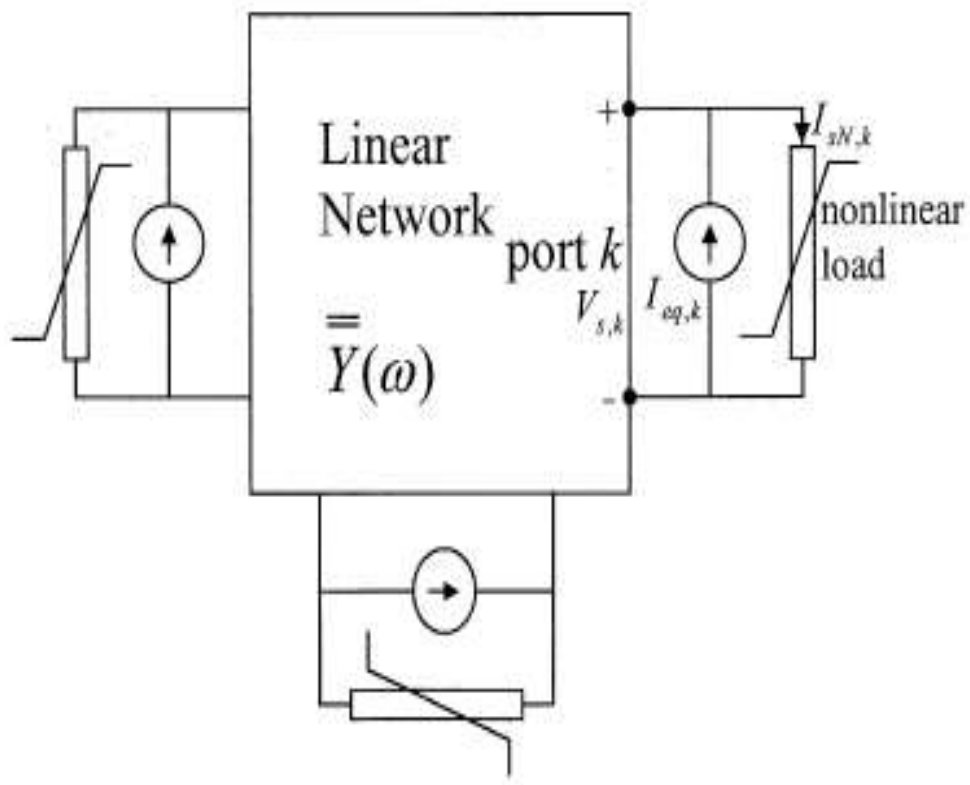

Figure 7: equivalent circuit of antenna array

In case the number of array antenna is $\mathrm{N}$, admittance matrix will be as follows, and in this research, we will use an antenna array $21 \times 21$ :

$$
\overline{\bar{Y}}=\left(\begin{array}{ccc}
\overline{\overline{Y_{1}}} & \ldots & \overline{\overline{Y_{1 N}}} \\
\vdots & \ddots & \vdots \\
\overline{\bar{Y}_{N 1}} & \cdots & \overline{\bar{Y}_{N N}}
\end{array}\right)
$$

$Y_{i i}$ and $Y_{i j}$ between antenna $i^{\text {th }}$ and $j^{\text {th }}$ are respectively insider admittance and alternate admittance which depends on the distance between antennas. At first, antennas are placed with 1 meter distance of each other.

Two poles of voltage rate diagram in antenna array for different frequencies is achieved as follows:

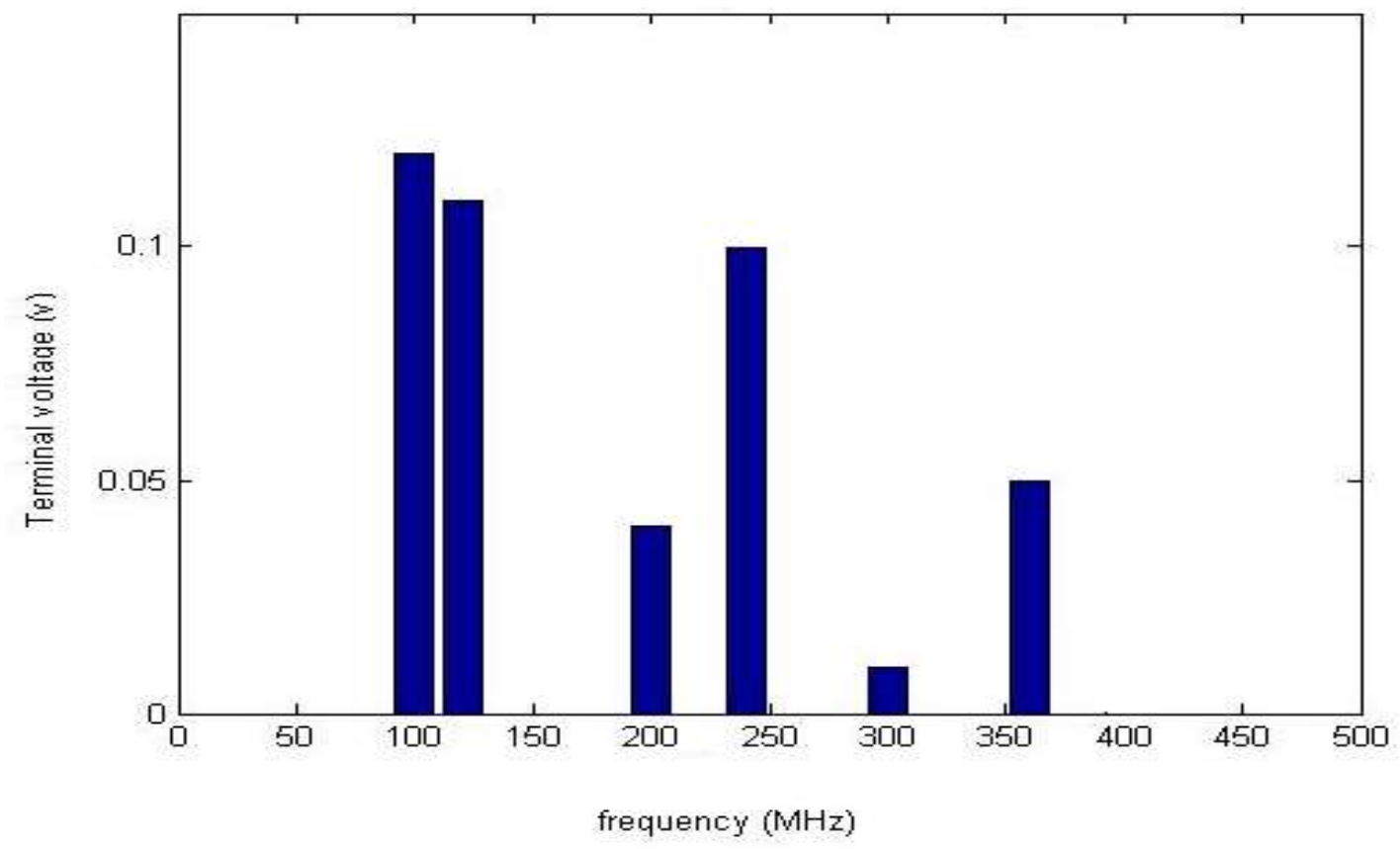

Figure 8: two poles of load in antenna array for different frequency (distance $2 \mathrm{~m}$ ) 
After increasing the distance between antennas from $1 \mathrm{~m}$ to $2 \mathrm{~m}$, we will have:

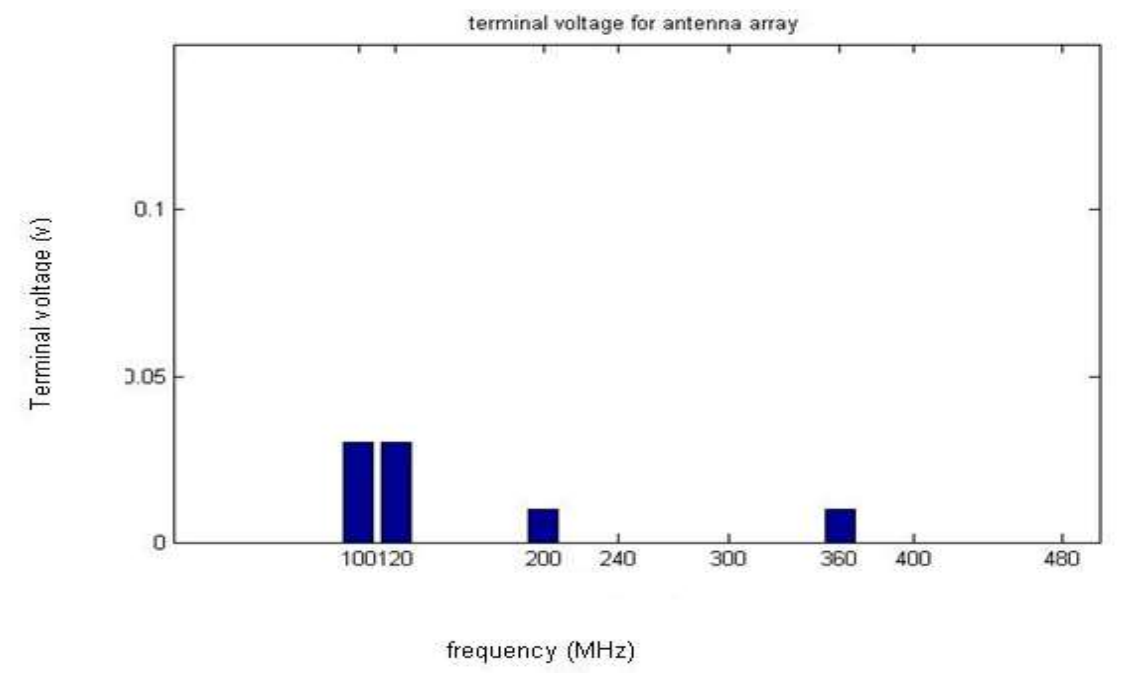

Figure 9: voltage of the two poles in antenna array for different frequencies (distance: $2 \mathrm{~m}$ )

We can see that after increasing the distance, some voltages are eliminated and some are attenuated, which is due to changes in admittance matrix $Y$. In fact, we were capable to analyze frequency selective, by introducing antenna array. Also, considering the relation $\bar{E}_{s}=\bar{E}_{i}+v \bar{E}_{r}$, and by having voltage charge of two poles, we can control the returning wave in different frequencies, by changing distance between antennas in antenna array.

It is worth noting that in introduced relation, $\bar{E}_{s}$ is returning wave. Also, $\bar{E}_{i}$ is reflected wave and $v$ is nonlinear voltage load and $\bar{E}_{i}$ is antenna radiation pattern.

Diagram of error attenuation algorithm for single antenna is as follows:

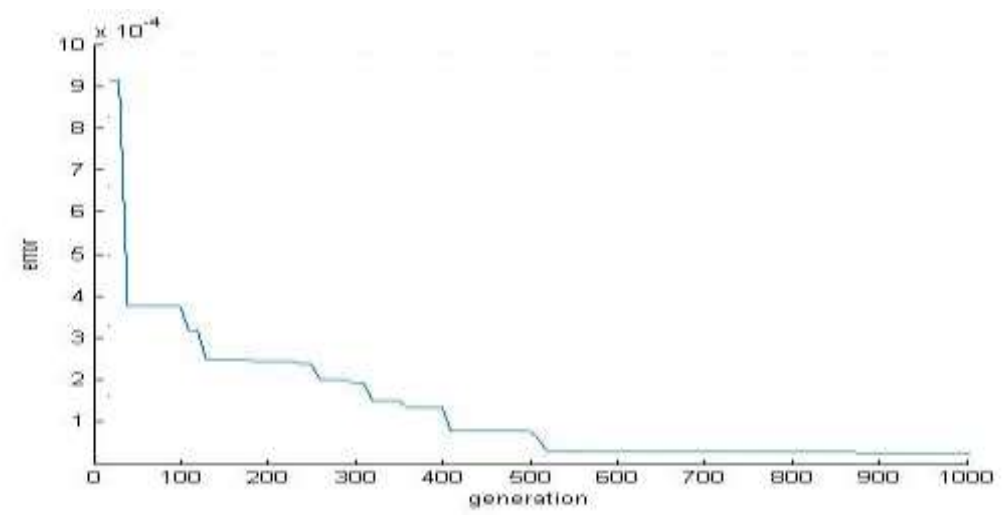

Figure 10: error attenuation algorithm diagram (single antenna)

Error attenuation algorithm for antenna array is as follows:

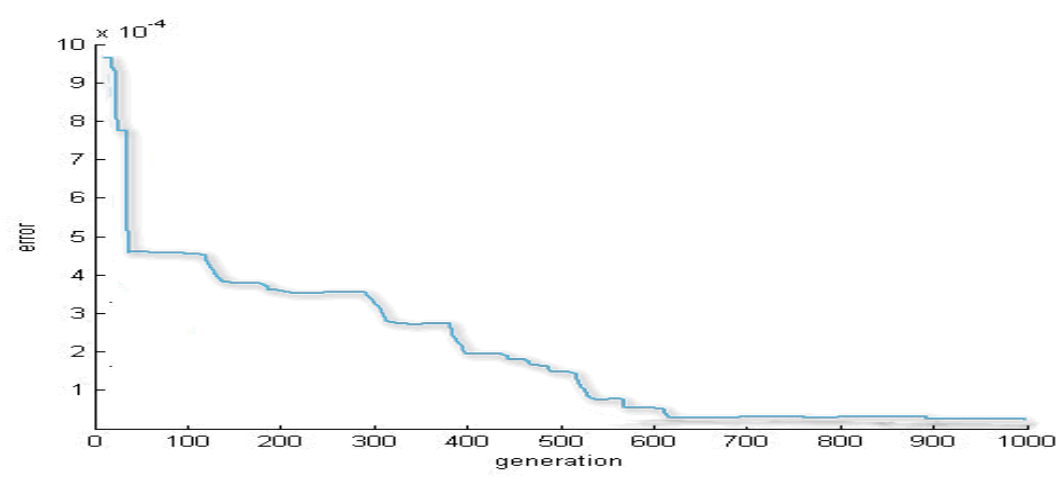

Figure 11: error attenuation algorithm (antenna array) diagram 
As we can see, in high iteration, algorithm error, is reached to its minimum value, and for single antenna, the error rate is a fix number from iteration 550 on, and for array, from iteration 610 on. Also, considering the figures, we have reached to convergence index, which is 0.0001 . In fact, by introducing this algorithm, we managed to analyze the structure of FSS.

\section{CONCLUSION}

Something that makes it necessary to address analysis of frequency selective surface, is to note that used methods in communication systems specially radars, have their own disadvantages, and removal of such disadvantages improves their social security, other than reduction of imposed expenses on countries; so, by taking into account economic and social conditions of our time, studying this field in order to reduce its disadvantages is necessary. In this study, after applying genetic algorithm, voltage of two nonlinear poles, for different harmonic is attenuated or reached to zero. This practice in radars decreases the probability of detection of the goal, and therefore, increases the security rate. Also, with respect to what we have already said, including the advantages of studied algorithm, we can mention to the possibility of the radiation of two frequencies instead of one. So, due to the fact that, in similar proposed methods for analysis of selective surfaces, as harmonic balance which can only radiate the single frequencies, this algorithm, presents more global analysis for frequency selective surface. Therefore, we can claim that the analysis presented in this research, is appropriate strategy for nonlinear issues and nonlinear loading carried out for antennas.

\section{References}

[1] Huang, C. C., \& Chu, T. H. (1993). Analysis of wire scatterers with nonlinear or time-harmonic loads in the frequency domain. Antennas and Propagation, IEEE Transactions on, 41(1), 25-30.

[2] Zhou, S., Li, W., \& Li, Q. (2010). Level-set based topology optimization for electromagnetic dipole antenna design. Journal of Computational Physics,229(19), 6915-6930.

[3] Razzak, M. A. (2015). A simple harmonic balance method for solving strongly nonlinear oscillators. Journal of the Association of Arab Universities for Basic and Applied Sciences.

[4] Ostadzadeh, S. R., Tayarani, M., \& Soleimani, M. (2009). A hybrid model in analyzing nonlinearly loaded dipole antenna and finite antenna array in the frequency domain. International journal of RF and microwave computeraided engineering, 19(4), 512.

[5] Feng, B., Li, S., An, W., Hong, W., Wang, S., \& Yin, S. (2014). A printed dual-wideband magneto-electric dipole antenna for WWAN/LTE applications.AEU-International Journal of Electronics and Communications, 68(10), 926932.

[6] Goswami, B., \& Mandal, D. (2013). A genetic algorithm for the level control of nulls and side lobes in linear antenna arrays. Journal of King Saud University-Computer and Information Sciences, 25(2), 117-126.

[7] Saxena, N. K., Khan, M. A., Pourush, P. K. S., \& Kumar, N. (2011). GA optimization of cutoff frequency of magnetically biased microstrip circular patch antenna. AEU-International Journal of Electronics and Communications, 65(5), 476-479.

[8] Mandal, K., \& Sarkar, P. P. (2015). A compact low profile wideband U-shape antenna with slotted circular ground plane. AEU-International Journal of Electronics and Communications.

[9] Lee, K. C. (2000). Analysis of large nonlinearly loaded antenna arrays under multitone excitation. Microwave and Optical Technology Letters, 25(5), 319-323.

[10] Huang, C. C., \& Chu, T. H. (1993). Analysis of wire scatterers with nonlinear or time-harmonic loads in the frequency domain. Antennas and Propagation, IEEE Transactions on, 41(1), 25-30.

[11] Zhou, S., Li, W., \& Li, Q. (2010). Level-set based topology optimization for electromagnetic dipole antenna design. Journal of Computational Physics,229(19), 6915-6930.

[12] Razzak, M. A. (2015). A simple harmonic balance method for solving strongly nonlinear oscillators. Journal of the Association of Arab Universities for Basic and Applied Sciences.

[13] Ostadzadeh, S. R., Tayarani, M., \& Soleimani, M. (2009). A hybrid model in analyzing nonlinearly loaded dipole antenna and finite antenna array in the frequency domain. International journal of RF and microwave computeraided engineering, 19(4), 512.

[14] Feng, B., Li, S., An, W., Hong, W., Wang, S., \& Yin, S. (2014). A printed dual-wideband magneto-electric dipole antenna for WWAN/LTE applications.AEU-International Journal of Electronics and Communications, 68(10), 926932.

[15] Goswami, B., \& Mandal, D. (2013). A genetic algorithm for the level control of nulls and side lobes in linear antenna arrays. Journal of King Saud University-Computer and Information Sciences, 25(2), 117-126.

[16] Saxena, N. K., Khan, M. A., Pourush, P. K. S., \& Kumar, N. (2011). GA optimization of cutoff frequency of magnetically biased microstrip circular patch antenna. AEU-International Journal of Electronics and Communications, 65(5), 476-479.

[17] Mandal, K., \& Sarkar, P. P. (2015). A compact low profile wideband U-shape antenna with slotted circular ground plane. AEU-International Journal of Electronics and Communications.

[18] Lee, K. C. (2000). Analysis of large nonlinearly loaded antenna arrays under multitone excitation. Microwave and Optical Technology Letters, 25(5), 319-323. 